\title{
Pricing American Options with Uncertain Volatility through Stochastic Linear Complementarity Models
}

\author{
Kenji Hamatani and Masao Fukushima \\ Department of Applied Mathematics and Physics \\ Graduate School of Informatics \\ Kyoto University \\ Kyoto 606-8501, Japan
}

February 19, 2010

\begin{abstract}
We consider the problem of pricing American options with uncertain volatility and propose two deterministic formulations based on the expected value method and the expected residual minimization method for a stochastic complementarity problem. We give sufficient conditions that ensure the existence of a solution of those deterministic formulations. Furthermore we show numerical results and discuss the usefulness of the proposed approach.
\end{abstract}

Key Words: Option pricing - American option - Uncertain volatility - Stochastic linear complementarity problem

\section{Introduction}

A derivative is a financial instrument whose value depends on the value of underlying assets such as stock, bond, currency and rate of interest [16]. Derivatives may be used for speculation purpose, but they are usually used for hedging the risk of fluctuation of a commodity or an exchange. Option is a kind of derivatives; it is the right to buy or sell the underlying assets by a certain date for a certain price. A call option is the right to buy an asset for a certain price. A put option is the right to sell an asset for a certain price, and the price at which the asset can be bought or sold in an option contract is called the strike price. A European option can be exercised only at the end of its life. An American option can be exercised at any time during its life, and the end of a contract is called the expiration date. Using the Black-Scholes model [3], we can compute the prices of European options explicitly under some assumptions. On the other hand, since an American option is permitted to exercise at any time of its life, we have to decide whether or not to exercise it and need to compute its boundary. Hence, pricing American options is more complicated than pricing European options. In particular, we cannot express the prices of American options explicitly and hence we can obtain the prices only by numerical computation. The binomial lattice model, finite difference approximation, and Monte Carlo simulation are used for pricing American options. In the binomial lattice model, we divide the time from now to the expiration date and create a binomial lattice representation of the asset price. Then, 
by backward induction on the lattice, we compute the prices of American options [10]. In the finite difference approximation method, we approximate the partial differential equation or partial differential inequality that the asset follows, and formulate pricing options as a linear complementarity problem [5, 15]. In Monte Carlo simulation, by sampling random paths of the process of the asset, we calculate the mean of the sample payoff and discount the expected payoff $[4,20]$.

The prices of European options and American options are dependent on the asset price, the strike price, the expiration date, the risk-free rate, and the volatility of the asset price. The Black-Scholes model [3] assumes that these values are constant. Since we know the asset price and the strike price correctly and the contractor can decide the expiration date, these values are absolutely constant. Moreover, we can expect the risk-free rate easily by seeing the interest rate of the bank deposits or the national bonds. However, it is practically difficult to set the volatility as a constant value, because each expert has his own view for the volatility. Besides, even if we adopt a historical volatility, it may fluctuate according to the chosen period. In practice, traders work with what are known as implied volatility. The implied volatility is the value calculated backward using the asset price, the strike price, the expiration date, the risk-free rate, and the price of option observed in the real market. Traders buy options if the implied volatility is comparatively low and sell options if it is comparatively high.

Recently, there have been a number of works on pricing options which suppose the volatility is not a constant value in order to remedy the shortcoming of the Black-Scholes model. In most of those works, the volatility of the asset is assumed to be stochastic and its variance is assumed to follow a mean-reverting process that indicates its tendency to return to a long-term average. Such a model is called the stochastic volatility model. The stochastic volatility model [14] gives a closed-form formula for the prices of the corresponding European options. For American options with varying volatility, their prices are obtained by using Heston model [14] via Monte Carlo simulation $[8,22]$. However, the stochastic volatility model assumes that the volatility varies with time. So this model may not suit the situation where the volatility is constant until the expiration time but uncertain at the present time.

In this paper, we assume that the volatility itself follows some probability distribution such as normal distribution and propose the formulation for pricing American options through a stochastic linear complementarity model. The stochastic complementarity problem is the problem whose coefficients are random variables. Since there is in general no solution that satisfies the complementarity conditions for all realizations of the coefficient values simultaneously, some deterministic formulations are constructed. We propose two deterministic formulations for pricing American options with uncertain volatility through the expected value method [13] and the expected residual minimization method [6]. Moreover, by analyzing numerical results based on some criteria, we show the usefulness of the proposed approach.

This paper is organized as follows: In Section 2, we recall the Black-Scholes partial differential equation and formulate pricing American options as a linear complementarity problem. In Section 3, we describe the expected value method and the expected residual minimization method for stochastic complementarity problems. In Section 4, we present two formulations for pricing American options with uncertain volatility by means of the expected value method and the expected residual minimization method. In Section 5, we discuss conditions that ensure the existence of a solution of those formulations. Numerical results are reported and discussed in Section 6. Finally, Section 7 concludes the paper. 


\section{Pricing American options using linear complementarity mod- els}

In this section, after reviewing the Black-Scholes partial differential equation [21], we describe a linear complementarity formulation for pricing American options [15].

First we specify the model of asset prices. Let $S$ denote the asset price at time $t$. Consider a small time interval $\mathrm{d} t$, during which $S$ changes to $S+\mathrm{d} S$. We can write the corresponding return on the asset as $\mathrm{d} S / S$. The common model decomposes this return into two parts. One is a deterministic return like the return on money invested in a risk-free bank. It gives the contribution

$$
\mu \mathrm{d} t
$$

to the return $\mathrm{d} S / S$, where $\mu$ is a measure of the average rate of growth of the asset price. In this paper, $\mu$ is taken to be a constant. The second part is a random change in the asset price in response to external effects such as unexpected news. It adds the term

$$
\sigma \mathrm{d} X
$$

to the return $\mathrm{d} S / S$. Here $\sigma$ is the standard deviation of returns, called the volatility, and $\mathrm{d} X$ is a Wiener process. The Wiener process has the following properties:

- $\mathrm{d} X$ has a normal distribution,

- the mean of $\mathrm{d} X$ is zero,

- the variance of $\mathrm{d} X$ is $\mathrm{d} t$.

Putting (2.1) and (2.2) together, we obtain the stochastic differential equation

$$
\frac{\mathrm{d} S}{S}=\mu \mathrm{d} t+\sigma \mathrm{d} X
$$

By multiplying both sides of the equation by $S$, we get the following equation:

$$
\mathrm{d} S=\mu S \mathrm{~d} t+\sigma S \mathrm{~d} X
$$

Now we recall the Black-Scholes partial differential equation, which is used for pricing European options. Throughout the paper, we make the following assumptions:

- The asset price follows the stochastic differential equation (2.3).

- There are no arbitrage possibilities. This means that there is no opportunity to make an instantaneous risk-free profit.

- Trading of the asset can take place continuously.

- Short selling is permitted and the asset is divisible. This means that we may sell assets that we do not own, and we can buy and sell any number (not necessarily an integer) of the asset. 
Let $V(S, t)$ denote the option price when the asset price is $S$ and the time is $t$. Then we can derive the following partial differential equation that $V(S, t)$ satisfies:

$$
\frac{\partial V}{\partial t}+\frac{1}{2} \sigma^{2} S^{2} \frac{\partial^{2} V}{\partial S^{2}}+r S \frac{\partial V}{\partial S}-r V=0
$$

This is called the Black-Scholes partial differential equation.

In the remainder of this section, we describe the linear complementarity model for pricing American options, as formulated by Huang and Pang [15]. Since we can exercise American options at any time during the life of the option, we have to determine not only option prices but also, for each value of $S$, whether or not it should be exercised. This is what is known as a free boundary problem. Since it is difficult to deal with free boundary, we reformulate the problem in such a way as to eliminate any explicit dependence on the free boundary. We describe a linear complementarity formulation for American option pricing.

Since a holder of American options may miss the optimal exercise price, there are cases where the portfolio consisting of American options cannot bring as high profit as the money invested in a bank. Moreover, by the assumption of no arbitrage possibilities, we cannot make a guaranteed riskless profit by borrowing money from the bank and investing in the portfolio. These observations yield, instead of the Black-Scholes partial differential equation, the following Black-Scholes partial differential inequality:

$$
\frac{\partial V}{\partial t}+\frac{1}{2} \sigma^{2} S^{2} \frac{\partial^{2} V}{\partial S^{2}}+r S \frac{\partial V}{\partial S}-r V \leq 0
$$

Let $\Lambda(S, t)$ denote the payoff function when the asset price is $S$ and the time is $t$. Payoff means the amount of money earned by exercising the right of options. For a call option, the payoff function is given by $\Lambda(S, t)=\max (S(t)-E, 0)$, where $E$ is the strike price. For a put option, the payoff function is given by $\Lambda(S, t)=\max (E-S(t), 0)$. If the price of an American option is less than the payoff, then an investor can earn the riskless profit by buying the option and immediately exercising it. Therefore, we must have

$$
V(S, t) \geq \Lambda(S, t)
$$

In addition, we have two choices for American options; we exercise the right of options or not. If we exercise, the price of an American option is equal to the payoff. If not, American options are essentially the same as European options. This means that the Black-Scholes partial differential equation (2.4) is valid. Thus, we obtain

$$
\left(\frac{\partial V}{\partial t}+\frac{1}{2} \sigma^{2} S^{2} \frac{\partial^{2} V}{\partial S^{2}}+r S \frac{\partial V}{\partial S}-r V\right)(V(S, t)-\Lambda(S, t))=0 .
$$

Putting (2.5), (2.6) and (2.7) together, we conclude that the prices of American options satisfy the partial differential complementarity condition:

$$
\begin{aligned}
& \frac{\partial V}{\partial t}+\frac{1}{2} \sigma^{2} S^{2} \frac{\partial^{2} V}{\partial S^{2}}+r S \frac{\partial V}{\partial S}-r V \leq 0 \\
& V(S, t)-\Lambda(S, t) \geq 0 \\
& \left(\frac{\partial V}{\partial t}+\frac{1}{2} \sigma^{2} S^{2} \frac{\partial^{2} V}{\partial S^{2}}+r S \frac{\partial V}{\partial S}-r V\right)(V(S, t)-\Lambda(S, t))=0 .
\end{aligned}
$$


Now we derive the (finite-dimensional) linear complementarity problem by discretizing the asset price and time. We divide the time interval $[0, T]$ into $L$ subintervals of equal length and denote

$$
t_{l}=l \delta t, \quad l=0,1,2, \cdots, L ; \quad \delta t=\frac{T}{L},
$$

where $T$ is the expiration date. The range of the asset price is $[0, \infty)$ in principle, but we assume that the actual asset price does not exceed a large positive number $S_{\max }$. We divide the interval $\left[0, S_{\max }\right]$ into $N$ subintervals of equal length and denote

$$
S_{n}=n \delta S, \quad n=1,2, \cdots, N ; \quad \delta S=\frac{S_{\max }}{N} .
$$

We write the discretized option prices and payoff values as follows:

$$
\left\{\begin{array}{c}
V_{n}^{l} \equiv V\left(S_{n}, t_{l}\right) \\
\Lambda_{n}^{l} \equiv \Lambda\left(S_{n}, t_{l}\right)
\end{array} \quad 1 \leq n \leq N, \quad 0 \leq l \leq L .\right.
$$

The partial differential complementarity problem (2.8) is then approximated on a regular grid with step-sizes $\delta t$ and $\delta S$. For the first partial derivative with respect to the time, we use the following forward difference approximation:

$$
\frac{\partial V}{\partial t}=\frac{V(S, t+\delta t)-V(S, t)}{\delta t}+\mathrm{O}(\delta t)
$$

For the first partial derivative with respect to the asset price, we use the following $\theta_{1}$-weighted central difference approximation:

$$
\begin{aligned}
\frac{\partial V}{\partial S}= & \theta_{1} \frac{V(S+\delta S, t)-V(S-\delta S, t)}{2 \delta S} \\
& +\left(1-\theta_{1}\right) \frac{V(S+\delta S, t+\delta t)-V(S-\delta S, t+\delta t)}{2 \delta S}+\mathrm{O}\left(\delta S^{2}\right),
\end{aligned}
$$

where $\theta_{1} \in[0,1]$ is a given parameter. When $\theta_{1}=0$, this approximation is called an explicit method. When $\theta_{1}=1$, this approximation is called an implicit method. When $\theta_{1}=1 / 2$, this approximation is called the Crank-Nicolson method. For the second partial derivative with respect to the asset price, we use the following $\theta_{2}$-weighted central difference approximation:

$$
\begin{aligned}
\frac{\partial^{2} V}{\partial S^{2}}= & \theta_{2} \frac{V(S+\delta S, t)-2 V(S, t)+V(S-\delta S, t)}{(\delta S)^{2}} \\
& +\left(1-\theta_{2}\right) \frac{V(S+\delta S, t+\delta t)-2 V(S, t+\delta t)+V(S-\delta S, t+\delta t)}{(\delta S)^{2}}+\mathrm{O}\left(\delta S^{2}\right),
\end{aligned}
$$

where $\theta_{2} \in[0,1]$ is a given parameter whose role is similar to that of $\theta_{1}$.

Using the difference approximations (2.12), (2.13) and (2.14), the left-hand side of the BlackScholes partial differential inequality can be approximated as follows: 


$$
\begin{aligned}
-\frac{\partial V}{\partial t}-r S \frac{\partial V}{\partial S}+r V-\frac{1}{2} \sigma^{2} S^{2} \frac{\partial^{2} V}{\partial S^{2}} \approx & V(S+\delta S, t)\left(-r S \theta_{1} \frac{1}{2 \delta S}-\frac{1}{2} \sigma^{2} S^{2} \theta_{2} \frac{1}{(\delta S)^{2}}\right) \\
& +V(S, t)\left(\frac{1}{\delta t}+r+\sigma^{2} S^{2} \theta_{2} \frac{1}{(\delta S)^{2}}\right) \\
& +V(S-\delta S, t)\left(-\frac{1}{2} \sigma^{2} S^{2} \theta_{2} \frac{1}{(\delta S)^{2}}+r S \theta_{1} \frac{1}{2 \delta S}\right) \\
& +V(S+\delta S, t+\delta t)\left(-r S\left(1-\theta_{1}\right) \frac{1}{2 \delta S}-\frac{1}{2} \sigma^{2} S^{2}\left(1-\theta_{2}\right) \frac{1}{(\delta S)^{2}}\right) \\
& +V(S, t+\delta t)\left(-\frac{1}{\delta t}+\sigma^{2} S^{2}\left(1-\theta_{2}\right) \frac{1}{(\delta S)^{2}}\right) \\
& +V(S-\delta S, t+\delta t)\left(-\frac{1}{2} \sigma^{2} S^{2}\left(1-\theta_{2}\right) \frac{1}{(\delta S)^{2}}+r S\left(1-\theta_{1}\right) \frac{1}{2 \delta S}\right) .
\end{aligned}
$$

With the above finite difference approximations, the system (2.8) leads to the following finitedimensional linear complementarity problem:

$$
0 \leq\left(\mathbf{V}^{l}-\mathbf{\Lambda}^{l}\right) \perp\left(\mathbf{M} \mathbf{V}^{l}+\mathbf{M}^{\prime} \mathbf{V}^{l+1}\right) \geq 0, \quad l=L-1, L-2, \cdots, 1,0,
$$

where the perp symbol $\perp$ denotes the orthogonality of two vectors, i.e., $x \perp y$ means $x^{T} y=0$, $\mathbf{V}^{l}$ and $\Lambda^{l}$ are $N$-vectors defined by

$$
\mathbf{V}^{l} \equiv\left(\begin{array}{c}
V_{1}^{l} \\
\vdots \\
V_{N}^{l}
\end{array}\right), \quad \Lambda^{l} \equiv\left(\begin{array}{c}
\Lambda_{1}^{l} \\
\vdots \\
\Lambda_{N}^{l}
\end{array}\right)
$$

$\mathbf{M}$ is the $N \times N$ matrix

$$
\mathbf{M} \equiv\left(\begin{array}{cccccccc}
b_{1} & c_{1} & 0 & 0 & 0 & 0 & \cdots & 0 \\
a_{2} & b_{2} & c_{2} & 0 & 0 & 0 & \cdots & 0 \\
0 & a_{3} & b_{3} & c_{3} & 0 & 0 & \cdots & 0 \\
& & & & & & & \\
\vdots & & & \ddots & \ddots & \ddots & & \vdots \\
& & & & & & & \\
0 & 0 & \cdots & 0 & 0 & a_{N-1} & b_{N-1} & c_{N-1} \\
0 & 0 & \cdots & 0 & 0 & 0 & a_{N} & b_{N}
\end{array}\right)
$$

with entries given by

$$
\begin{array}{rlrl}
a_{n} & =-\frac{1}{2} \sigma^{2} n^{2} \theta_{2}+\frac{r n \theta_{1}}{2}, & & n=2, \cdots, N \\
b_{n}=r+\frac{1}{\delta t}+\sigma^{2} n^{2} \theta_{2}, & n & =1, \cdots, N \\
c_{n}=-\frac{r n \theta_{1}}{2}-\frac{1}{2} \sigma^{2} n^{2} \theta_{2}, & n=1, \cdots, N-1,
\end{array}
$$

and $\mathbf{M}^{\prime}$ is the $N \times N$ matrix, formed in the same way as $\mathbf{M}$, with entries given by

$$
\begin{array}{rlrl}
a_{n}^{\prime}=-\frac{1}{2} \sigma^{2} n^{2}\left(1-\theta_{2}\right)+\frac{r n\left(1-\theta_{1}\right)}{2}, & n=2, \cdots, N \\
b_{n}^{\prime}=-\frac{1}{\delta t}+\sigma^{2} n^{2}\left(1-\theta_{2}\right), & n=1, \cdots, N & \\
c_{n}^{\prime}=-\frac{r n\left(1-\theta_{1}\right)}{2}-\frac{1}{2} \sigma^{2} n^{2}\left(1-\theta_{2}\right), & n=1, \cdots, N-1 .
\end{array}
$$


On the expiration date, we cannot hold American options any more. So we have to exercise the right of options or discard it. This means that, on the expiration date, the price of an American option is equal to the payoff value, that is to say, $\mathbf{V}^{L}=\boldsymbol{\Lambda}^{L}$. Since $\mathbf{V}^{L}$ is known, we can solve the linear complementarity problems (2.15) for $l=L-1, L-2, \cdots, 1,0$, by proceeding backward in time. Thus, we can obtain a set of discrete option prices at $t=0$ as $V_{n}^{0}, n=1, \cdots, N$.

\section{Deterministic formulations for the stochastic complementar- ity problem}

In this section, we consider the general stochastic complementarity problem and describe the expected value method [13] and the expected residual minimization method [6] which give deterministic formulations for the stochastic complementarity problem.

The stochastic complementarity problem in standard form is to find a vector $x \in \Re_{+}^{n}$ such that

$$
0 \leq x \perp F(x, \omega) \geq 0, \quad \omega \in \Omega,
$$

where $F: \Re^{n} \times \Omega \rightarrow \Re^{n}$ is a vector-valued function, $(\Omega, \mathcal{F}, P)$ is a probability space with $\Omega \subseteq \Re^{m}$. In general, there is no vector $x \in \Re_{+}^{n}$ satisfying (3.1) for all $\omega \in \Omega$ simultaneously. Therefore, it is necessary to consider a deterministic formulation for (3.1) which provides an optimal solution of the stochastic complementarity problem in some sense.

\subsection{Expected value method}

The expected value method [13] considers the deterministic formulation which is to find a vector $x \in \Re_{+}^{n}$ such that

$$
0 \leq x \perp F_{\infty}(x) \geq 0,
$$

where $F_{\infty}(x):=\mathrm{E}[F(x, \omega)]$ is the expectation function of the random function $F(x, \omega)$. Since it is usually difficult to evaluate the expectation function $F_{\infty}(x)$ exactly, we use a finite number of samples $\left\{\omega_{j}, j=1, \cdots, k\right\}$ and construct an approximating function $F_{k}(x)$ as

$$
F_{k}(x):=\frac{1}{k} \sum_{j=1}^{k} F\left(x, \omega_{j}\right) .
$$

By using the approximating function $F_{k}(x)$, the complementarity problem (3.2) is rewritten as

$$
0 \leq x \perp F_{k}(x) \geq 0 \text {. }
$$

\subsection{Expected residual minimization method}

We consider a function $\psi: \Re^{2} \rightarrow \Re$, called an NCP function, which satisfies

$$
\psi(a, b)=0 \Longleftrightarrow a \geq 0, b \geq 0, a b=0 .
$$

There are various NCP functions for solving complementarity problems [12]. In this paper we concentrate on two popular NCP functions; the min function

$$
\psi(a, b)=\min (a, \nu b)
$$


and the Fischer-Burmeister (FB) function

$$
\psi(a, b)=a+\nu b-\sqrt{a^{2}+(\nu b)^{2}},
$$

where $\nu$ is a positive parameter. Then, we can easily verify that (3.1) is equivalent to the following equation:

$$
\Psi(x, \omega)=0, \quad \omega \in \Omega,
$$

where $\Psi: \Re^{n} \times \Omega \rightarrow \Re^{n}$ is defined by

$$
\Psi(x, \omega):=\left(\begin{array}{c}
\psi\left(F_{1}(x, \omega), x_{1}\right) \\
\vdots \\
\psi\left(F_{n}(x, \omega), x_{n}\right)
\end{array}\right) .
$$

As mentioned above, there is usually no $x \in \Re_{+}^{n}$ satisfying (3.6) for all $\omega \in \Omega$ simultaneously. In [6], the expected residual minimization method is proposed to give the following deterministic formulation for the stochastic complementarity problem:

$$
\begin{array}{ll}
\min _{x} & \mathrm{E}\left[\|\Psi(x, \omega)\|^{2}\right] \\
\text { s.t. } & x \in \Re_{+}^{n},
\end{array}
$$

where $\|\cdot\|$ denotes the Euclidean norm. Like the expected value method, it is usually difficult to evaluate the expectation $\mathrm{E}\left[\|\Psi(x, \omega)\|^{2}\right]$ exactly. So we use a finite number of samples $\left\{\omega_{j}, j=\right.$ $1, \cdots, k\}$ and construct an approximating function of $\mathrm{E}\left[\|\Psi(x, \omega)\|^{2}\right]$ as

$$
f_{k}(x):=\frac{1}{k} \sum_{j=1}^{k}\left\|\Psi\left(x, \omega_{j}\right)\right\|^{2} .
$$

By using the approximating function, problem (3.7) is rewritten as

$$
\begin{array}{ll}
\min _{x} & f_{k}(x) \\
\text { s.t. } & x \in \Re_{+}^{n} .
\end{array}
$$

This approach may be regarded as an extension of the least-squares method for an overdetermined system of equations.

We note that, if $\Omega$ has only one realization, then we get the same solution by using the expected value method (3.2) and the expected residual minimization method (3.7) as long as the original complementarity problem has a solution, and the solubility of the expected residual minimization method (3.7) does not depend on the choice of NCP functions. It should be noted, however, that we usually get different solutions by using the expected value method and the expected residual minimization method if $\Omega$ has more than one realization. Moreover, the solubility of the expected residual minimization method (3.7) is dependent on the choice of NCP functions [6]. In other words, a solution of the stochastic complementarity problem depends on the choice of deterministic formulations. Besides, there are cases where the solution set is empty or there are many solutions. In Section 5, we discuss conditions that ensure the existence of a solution in deterministic formulations for the stochastic linear complementarity problem derived from the model for pricing American options. 


\section{Pricing American options with uncertain volatility}

In this section, we present two deterministic formulations for pricing American options with uncertain volatility, which are based on the expected value method and the expected residual minimization method for the stochastic complementarity problem discussed in Section 3. Since the entries of the matrices $\mathbf{M}$ and $\mathbf{M}^{\prime}$ defined in Section 2 are dependent on the volatility $\sigma$, we write $\mathbf{M}(\sigma)$ and $\mathbf{M}^{\prime}(\sigma)$.

If we regard the volatility $\sigma$ as a random variable, pricing American options with uncertain volatility is formulated as the following stochastic linear complementarity problem:

$$
0 \leq\left(\mathbf{V}^{l}-\mathbf{\Lambda}^{l}\right) \perp\left(\mathbf{M}(\sigma) \mathbf{V}^{l}+\mathbf{M}^{\prime}(\sigma) \mathbf{V}^{l+1}\right) \geq 0, \quad l=L-1, L-2, \cdots, 1,0 .
$$

As mentioned in Section 3, there are usually no $V^{l}, l=0,1, \cdots, L-1$ satisfying (4.1) for all $\sigma$ simultaneously. So we apply the expected value method and the expected residual minimization method to the stochastic linear complementarity problem (4.1).

First, we give the formulation based on the expected value method. In the expected value method, we substitute the expected values $\mathrm{E}[\mathbf{M}(\sigma)]$ and $\mathrm{E}\left[\mathbf{M}^{\prime}(\sigma)\right]$ for $\mathbf{M}(\sigma)$ and $\mathbf{M}^{\prime}(\sigma)$, respectively. Then we have the following linear complementarity problem:

$$
0 \leq\left(\mathbf{V}^{l}-\mathbf{\Lambda}^{l}\right) \perp\left(\mathrm{E}[\mathbf{M}(\sigma)] \mathbf{V}^{l}+\mathrm{E}\left[\mathbf{M}^{\prime}(\sigma)\right] \mathbf{V}^{l+1}\right) \geq 0, \quad l=L-1, L-2, \cdots, 1,0 .
$$

Using discrete samples $\left\{\sigma_{j}, j=1, \cdots, k\right\}$, the expected values $\mathrm{E}[\mathbf{M}(\sigma)]$ and $\mathrm{E}\left[\mathbf{M}^{\prime}(\sigma)\right]$ can be approximated by $\frac{1}{k} \sum_{j=1}^{k} \mathbf{M}\left(\sigma_{j}\right)$ and $\frac{1}{k} \sum_{j=1}^{k} \mathbf{M}^{\prime}\left(\sigma_{j}\right)$, respectively. So (4.2) can be rewritten as

$$
0 \leq\left(\mathbf{V}^{l}-\mathbf{\Lambda}^{l}\right) \perp\left(\frac{1}{k} \sum_{j=1}^{k} \mathbf{M}\left(\sigma_{j}\right) \mathbf{V}^{l}+\frac{1}{k} \sum_{j=1}^{k} \mathbf{M}^{\prime}\left(\sigma_{j}\right) \mathbf{V}^{l+1}\right) \geq 0, \quad l=L-1, L-2, \cdots, 1,0 .
$$

Like pricing American options with constant volatility, the price of an option on the expiration date is equal to the payoff value, that is $\mathbf{V}^{L}=\boldsymbol{\Lambda}^{L}$. By solving (4.3) backward in time, we can obtain a set of discrete option prices at $t=0$ as $V_{n}^{0}, n=1, \cdots, N$.

Next, we give the formulation based on the expected residual minimization method. Using the equality $\mathbf{V}^{L}=\boldsymbol{\Lambda}^{L}$, the stochastic linear complementarity problem (4.1) can be rewritten as the following stochastic linear complementarity problem:

$$
0 \leq\left(\begin{array}{c}
\mathbf{V}^{0}-\boldsymbol{\Lambda}^{0} \\
\mathbf{V}^{1}-\boldsymbol{\Lambda}^{1} \\
\vdots \\
\mathbf{V}^{L-2}-\boldsymbol{\Lambda}^{L-2} \\
\mathbf{V}^{L-1}-\boldsymbol{\Lambda}^{L-1}
\end{array}\right) \perp\left(\begin{array}{cccccc}
\mathbf{M}(\sigma) & \mathbf{M}^{\prime}(\sigma) & 0 & 0 & \cdots & 0 \\
0 & \mathbf{M}(\sigma) & \mathbf{M}^{\prime}(\sigma) & 0 & \cdots & 0 \\
\vdots & & \ddots & \ddots & \ddots & \vdots \\
0 & 0 & \cdots & 0 & \mathbf{M}(\sigma) & \mathbf{M}^{\prime}(\sigma) \\
0 & 0 & \cdots & 0 & 0 & \mathbf{M}(\sigma)
\end{array}\right)\left(\begin{array}{c}
\mathbf{V}^{0} \\
\mathbf{V}^{1} \\
\\
\vdots \\
\mathbf{V}^{L-2} \\
\mathbf{V}^{L-1}
\end{array}\right)+\left(\begin{array}{c}
0 \\
0 \\
\vdots \\
\\
0 \\
\mathbf{M}^{\prime}(\sigma) \boldsymbol{\Lambda}^{L}
\end{array}\right) \geq 0
$$


where $\mathbf{V}^{l}, l=0,1, \cdots, L-1$ are the variables. We define $\mathbf{V}$ and $\Psi(\mathbf{V}, \sigma)$ as

$$
\mathbf{V}=\left(\begin{array}{c}
\mathbf{V}^{0} \\
\mathbf{V}^{1} \\
\vdots \\
\mathbf{V}^{L-2} \\
\mathbf{V}^{L-1}
\end{array}\right), \quad \Psi(\mathbf{V}, \sigma)=\left(\begin{array}{c}
\psi\left(V_{1}^{0}-\Lambda_{1}^{0},\left(\mathbf{M}(\sigma) \mathbf{V}^{0}+\mathbf{M}^{\prime}(\sigma) \mathbf{V}^{1}\right)_{1}\right) \\
\vdots \\
\psi\left(V_{N}^{0}-\Lambda_{N}^{0},\left(\mathbf{M}(\sigma) \mathbf{V}^{0}+\mathbf{M}^{\prime}(\sigma) \mathbf{V}^{1}\right)_{N}\right) \\
\psi\left(V_{1}^{1}-\Lambda_{1}^{1},\left(\mathbf{M}(\sigma) \mathbf{V}^{1}+\mathbf{M}^{\prime}(\sigma) \mathbf{V}^{2}\right)_{1}\right) \\
\vdots \\
\psi\left(V_{N}^{1}-\Lambda_{N}^{1},\left(\mathbf{M}(\sigma) \mathbf{V}^{1}+\mathbf{M}^{\prime}(\sigma) \mathbf{V}^{2}\right)_{N}\right) \\
\vdots \\
\psi\left(V_{1}^{L-1}-\Lambda_{1}^{L-1},\left(\mathbf{M}(\sigma) \mathbf{V}^{L-1}+\mathbf{M}^{\prime}(\sigma) \mathbf{V}^{L}\right)_{1}\right) \\
\vdots \\
\psi\left(V_{N}^{L-1}-\Lambda_{N}^{L-1},\left(\mathbf{M}(\sigma) \mathbf{V}^{L-1}+\mathbf{M}^{\prime}(\sigma) \mathbf{V}^{L}\right)_{N}\right)
\end{array}\right),
$$

where $\psi$ is an NCP function and $\left(\mathbf{M}(\sigma) \mathbf{V}^{l}+\mathbf{M}^{\prime}(\sigma) \mathbf{V}^{l+1}\right)_{n}$ denotes the $n$th component of the vector $\mathbf{M}(\sigma) \mathbf{V}^{l}+\mathbf{M}^{\prime}(\sigma) \mathbf{V}^{l+1}$.

Using the expected residual minimization method, pricing American options with uncertain volatility is formulated as the following optimization problem:

$$
\begin{array}{ll}
\min _{\mathbf{V}} & \mathrm{E}\left[\|\Psi(\mathbf{V}, \sigma)\|^{2}\right] \\
\text { s.t. } & \mathbf{V}^{l} \geq \boldsymbol{\Lambda}^{l}, \quad l=0,1, \cdots, L-1, \\
& \mathbf{V}^{L}=\boldsymbol{\Lambda}^{L} .
\end{array}
$$

Adopting the min function as the NCP function $\psi,(4.5)$ can be rewritten as

$$
\begin{array}{ll}
\min _{\mathbf{V}} & \mathrm{E}\left[\sum_{l=0}^{L-1} \sum_{n=1}^{N}\left\{\min \left(V_{n}^{l}-\Lambda_{n}^{l}, \nu\left(\mathbf{M}(\sigma) \mathbf{V}^{l}+\mathbf{M}^{\prime}(\sigma) \mathbf{V}^{l+1}\right){ }_{n}\right)\right\}^{2}\right] \\
\text { s.t. } & \mathbf{V}^{l} \geq \mathbf{\Lambda}^{l}, \quad l=0,1, \cdots, L-1, \\
& \mathbf{V}^{L}=\boldsymbol{\Lambda}^{L} .
\end{array}
$$

Moreover, by using discrete samples $\left\{\sigma_{j}, j=1, \cdots, k\right\}$, (4.6) can be approximated as follows:

$$
\begin{array}{ll}
\min _{\mathbf{V}} & \frac{1}{k} \sum_{j=1}^{k} \sum_{l=0}^{L-1} \sum_{n=1}^{N}\left\{\min \left(V_{n}^{l}-\Lambda_{n}^{l}, \nu\left(\mathbf{M}\left(\sigma_{j}\right) \mathbf{V}^{l}+\mathbf{M}^{\prime}\left(\sigma_{j}\right) \mathbf{V}^{l+1}\right)_{n}\right)\right\}^{2} \\
\text { s.t. } & \mathbf{V}^{l} \geq \boldsymbol{\Lambda}^{l}, \quad l=0,1, \cdots, L-1, \\
& \mathbf{V}^{L}=\boldsymbol{\Lambda}^{L} .
\end{array}
$$

\section{Choice of step-size parameter and existence of a solution}

In this section, we give conditions that ensure the existence of a solution in the formulation by the expected value method (4.3) and the formulation by the expected residual minimization method (4.3) for pricing American options with uncertain volatility. Recall that we can take the step-size parameter $\delta t$ arbitrarily for a certain positive integer $L$ satisfying (2.9). So we mainly examine conditions for the parameter $\delta t$ that ensure the existence of a solution. 


\subsection{Existence of a solution in the expected value method}

We denote the discrete samples of $\sigma$ as $\left\{\sigma_{j}, j=1, \cdots, k\right\}$. Then, the coefficient matrix of the linear complementarity problem in the expected value method (4.3) is written as

$$
\tilde{\mathbf{M}} \equiv\left(\begin{array}{cccccccc}
\tilde{b}_{1} & \tilde{c}_{1} & 0 & 0 & 0 & 0 & \ldots & 0 \\
\tilde{a}_{2} & \tilde{b}_{2} & \tilde{c}_{2} & 0 & 0 & 0 & \ldots & 0 \\
0 & \tilde{a}_{3} & \tilde{b}_{3} & \tilde{c}_{3} & 0 & 0 & \ldots & 0 \\
& & & & & & & \\
\vdots & & & \ddots & \ddots & \ddots & & \vdots \\
& & & & & & & \\
0 & 0 & \cdots & 0 & 0 & \tilde{a}_{N-1} & \tilde{b}_{N-1} & \tilde{c}_{N-1} \\
0 & 0 & \cdots & 0 & 0 & 0 & \tilde{a}_{N} & \tilde{b}_{N}
\end{array}\right)
$$

with entries given by

$$
\begin{aligned}
& \tilde{a}_{n}=-\frac{n^{2} \theta_{2}}{2 k} \sum_{j=1}^{k} \sigma_{j}^{2}+\frac{r n \theta_{1}}{2}, \quad n=2, \cdots, N \\
& \tilde{b}_{n}=r+\frac{1}{\delta t}+\frac{n^{2} \theta_{2}}{k} \sum_{j=1}^{k} \sigma_{j}^{2}, \quad n=1, \cdots, N \\
& \tilde{c}_{n}=-\frac{r n \theta_{1}}{2}-\frac{n^{2} \theta_{2}}{2 k} \sum_{j=1}^{k} \sigma_{j}^{2}, \quad n=1, \cdots, N-1 .
\end{aligned}
$$

For a square matrix $A \in \Re^{n \times n}$, the following results are known [19].

Lemma 1. If a square matrix $A$ is a strictly row diagonally dominant matrix with positive diagonal elements, then $A$ is a P-matrix.

Recall that $A=\left(a_{i j}\right)$ is said to be strictly row diagonally dominant if

$$
\left|a_{i i}\right|>\sum_{j \neq i}\left|a_{i j}\right|, \quad i=1, \cdots, n .
$$

A square matrix is said to be a P-matrix if all its principal minors are positive. About a P-matrix, the following results are known [9].

Lemma 2. Let $A \in \Re^{n \times n}$. Then the following statement are equivalent:

(a) A is a P-matrix.

(b) Matrix A reverses the sign of no vector, i.e.,

$$
x_{i}(A x)_{i} \leq 0, \quad \forall i \quad \Rightarrow \quad x=0 .
$$

(c) the linear complementarity problem

$$
0 \leq x \perp A x+q \geq 0
$$

has a unique solution for any vector $q \in \Re^{n}$.

Concerning the choice of $\delta t$, we can establish the following proposition. 
Proposition 1. If we choose $\delta t$ such that

$$
\frac{1}{\delta t}>\frac{k r^{2} \theta_{1}^{2}}{4 \theta_{2} \sum_{j=1}^{k} \sigma_{j}^{2}}-r
$$

then the linear complementarity problem (4.3) in the expected value method has a unique solution.

Proof. Clearly, all diagonal elements of $\tilde{\mathbf{M}}$ are positive. We will prove that $\tilde{\mathbf{M}}$ is a strictly row diagonally dominant matrix. Note that $\tilde{\mathbf{M}}$ is a strictly row diagonally dominant if and only if

$$
\begin{aligned}
& \left|\tilde{b}_{1}\right|>\left|\tilde{c}_{1}\right|, \\
& \left|\tilde{b}_{n}\right|>\left|\tilde{a}_{n}\right|+\left|\tilde{c}_{n}\right|, \quad n=2, \cdots, N-1, \\
& \left|\tilde{b}_{N}\right|>\left|\tilde{a}_{N}\right| .
\end{aligned}
$$

Since $\tilde{b}_{n}, n=1, \cdots, N$ are positive and $\tilde{c}_{n}, n=1, \cdots, N-1$ are negative, we can write

$$
\begin{aligned}
\left|\tilde{b}_{1}\right|-\left|\tilde{c}_{1}\right|= & r+\frac{1}{\delta t}+\frac{\theta_{2}}{k} \sum_{j=1}^{k} \sigma_{j}^{2}-\frac{r \theta_{1}}{2}-\frac{\theta_{2}}{2 k} \sum_{j=1}^{k} \sigma_{j}^{2}, \\
\left|\tilde{b}_{n}\right|-\left|\tilde{a}_{n}\right|-\left|\tilde{c}_{n}\right|= & r+\frac{1}{\delta t}+\frac{n^{2} \theta_{2}}{k} \sum_{j=1}^{k} \sigma_{j}^{2}-\left|-\frac{n^{2} \theta_{2}}{2 k} \sum_{j=1}^{k} \sigma_{j}^{2}+\frac{r n \theta_{1}}{2}\right| \\
& -\frac{r n \theta_{1}}{2}-\frac{n^{2} \theta_{2}}{2 k} \sum_{j=1}^{k} \sigma_{j}^{2}, \quad n=2, \cdots, N-1, \\
\left|\tilde{b}_{N}\right|-\left|\tilde{a}_{N}\right|= & r+\frac{1}{\delta t}+\frac{N^{2} \theta_{2}}{k} \sum_{j=1}^{k} \sigma_{j}^{2}-\left|-\frac{N^{2} \theta_{2}}{2 k} \sum_{j=1}^{k} \sigma_{j}^{2}+\frac{r N \theta_{1}}{2}\right| .
\end{aligned}
$$

We only consider the cases of $n=2, \cdots, N-1$, because the cases $n=1$ and $n=N$ can be treated similarly. First, suppose $a_{n} \geq 0$. Then we can write

$$
\left|\tilde{b}_{n}\right|-\left|\tilde{a}_{n}\right|-\left|\tilde{c}_{n}\right|=r+\frac{1}{\delta t}+\frac{n^{2} \theta_{2}}{k} \sum_{j=1}^{k} \sigma_{j}^{2}-r n \theta_{1}, \quad n=2, \cdots, N-1 .
$$

Note that the right-hand of (5.3) can be rewritten as

$$
\frac{\theta_{2}}{k} \sum_{j=1}^{k} \sigma_{j}^{2}\left(n-\frac{k r \theta_{1}}{2 \theta_{2} \sum_{j=1}^{k} \sigma_{j}^{2}}\right)^{2}+\frac{1}{\delta t}-\frac{k r^{2} \theta_{1}^{2}}{4 \theta_{2} \sum_{j=1}^{k} \sigma_{j}^{2}}+r, \quad n=2, \cdots, N-1 .
$$

Hence if $\delta t$ satisfies (5.1), we have (5.2).

Next, suppose $a_{n}<0$. Then we can write

$$
\left|\tilde{b}_{n}\right|-\left|\tilde{a}_{n}\right|-\left|\tilde{c}_{n}\right|=r+\frac{1}{\delta t}, \quad n=2, \cdots, N-1 .
$$

Since $r \geq 0$ and $\delta t>0$, we have (5.2).

Therefore, if $\delta t$ is chosen to satisfy (5.1), then $\tilde{\mathbf{M}}$ is a strictly row diagonally dominant matrix. By Lemma 1, this implies that $\tilde{\mathbf{M}}$ is a P-matrix. Then the assertion of the proposition follows from Lemma 2 . 


\subsection{Existence of a solution in the expected residual minimization method}

Next, we examine conditions that ensure the existence of a solution in the expected residual minimization method. We denote the discrete samples of $\sigma$ as $\left\{\sigma_{j}, j=1, \cdots, k\right\}$. For each $\sigma_{j}$, the coefficient matrix (4.4) is written as

$$
\mathbf{G}\left(\sigma_{j}\right)=\left(\begin{array}{cccccc}
\hat{\mathbf{M}}\left(\sigma_{j}\right) & \hat{\mathbf{M}}^{\prime}\left(\sigma_{j}\right) & 0 & 0 & \ldots & 0 \\
0 & \hat{\mathbf{M}}\left(\sigma_{j}\right) & \hat{\mathbf{M}}^{\prime}\left(\sigma_{j}\right) & 0 & \ldots & 0 \\
\vdots & & \ddots & \ddots & \ddots & \vdots \\
0 & 0 & \ldots & 0 & \hat{\mathbf{M}}\left(\sigma_{j}\right) & \hat{\mathbf{M}}^{\prime}\left(\sigma_{j}\right) \\
0 & 0 & \ldots & 0 & 0 & \hat{\mathbf{M}}\left(\sigma_{j}\right)
\end{array}\right) \text {, }
$$

where $\hat{\mathbf{M}}\left(\sigma_{j}\right)$ is the $N \times N$ matrix

$$
\hat{\mathbf{M}}\left(\sigma_{j}\right)=\left(\begin{array}{cccccccc}
\hat{b}_{1} & \hat{c}_{1} & 0 & 0 & 0 & 0 & \cdots & 0 \\
\hat{a}_{2} & \hat{b}_{2} & \hat{c}_{2} & 0 & 0 & 0 & \cdots & 0 \\
0 & \hat{a}_{3} & \hat{b}_{3} & \hat{c}_{3} & 0 & 0 & \cdots & 0 \\
& & & & & & & \\
\vdots & & & \ddots & \ddots & \ddots & & \vdots \\
& & & & & & & \\
0 & 0 & \cdots & 0 & 0 & \hat{a}_{N-1} & \hat{b}_{N-1} & \hat{c}_{N-1} \\
0 & 0 & \cdots & 0 & 0 & 0 & \hat{a}_{N} & \hat{b}_{N}
\end{array}\right)
$$

with entries given by

$$
\begin{aligned}
& \hat{a}_{n}=-\frac{1}{2} \sigma_{j}^{2} n^{2} \theta_{2}+\frac{r n \theta_{1}}{2}, \quad n=2, \cdots, N \\
& \hat{b}_{n}=r+\frac{1}{\delta t}+\sigma_{j}^{2} n^{2} \theta_{2}, \quad n=1, \cdots, N \\
& \hat{c}_{n}=-\frac{r n \theta_{1}}{2}-\frac{1}{2} \sigma_{j}^{2} n^{2} \theta_{2}, \quad n=1, \cdots, N-1,
\end{aligned}
$$

and $\hat{\mathbf{M}}^{\prime}\left(\sigma_{j}\right)$ is the $N \times N$ matrix, formed in the same way as $\hat{\mathbf{M}}\left(\sigma_{j}\right)$, with entries given by

$$
\begin{aligned}
& \hat{a}_{n}^{\prime}=-\frac{1}{2} \sigma_{j}^{2} n^{2}\left(1-\theta_{2}\right)+\frac{r n\left(1-\theta_{1}\right)}{2}, \quad n=2, \cdots, N \\
& \hat{b}_{n}^{\prime}=-\frac{1}{\delta t}+\sigma_{j}^{2} n^{2}\left(1-\theta_{2}\right), \quad n=1, \cdots, N \\
& \hat{c}_{n}^{\prime}=-\frac{r n\left(1-\theta_{1}\right)}{2}-\frac{1}{2} \sigma_{j}^{2} n^{2}\left(1-\theta_{2}\right), \quad n=1, \cdots, N-1 .
\end{aligned}
$$

Recall that a square matrix $H$ is called an $R_{0}$ matrix if

$$
x^{T} H x=0, H x \geq 0, x \geq 0 \Rightarrow x=0 .
$$

In particular, any $\mathrm{P}$-matrix is an $R_{0}$ matrix [9]. The following existence result has been established for the expected residual minimization method [6].

Lemma 3. If $\boldsymbol{G}\left(\sigma_{j}\right)$ is an $R_{0}$ matrix for some $j \in\{1, \cdots, k\}$, then the solution set of the optimization problem (4.7) is nonempty and bounded. 
Considering the choice of the parameter $\delta t$, we have the following proposition.

Proposition 2. If we choose $\delta t$ such that

$$
\frac{1}{\delta t}>\frac{r^{2} \theta_{1}^{2}}{4 \theta_{2} \sigma_{j}^{2}}-r
$$

for some $j \in\{1, \cdots, k\}$, then the solution set of the optimization problem (4.7) in the expected residual minimization method is nonempty and bounded.

Proof. In a similar manner to the proof of Proposition 1, we can verify that all diagonal elements of $\hat{\mathbf{M}}\left(\sigma_{j}\right)$ are positive and $\hat{\mathbf{M}}\left(\sigma_{j}\right)$ is a strictly row diagonally dominant matrix, whenever $\delta t$ satisfies (2). Therefore, $\hat{\mathbf{M}}\left(\sigma_{j}\right)$ is a P-matrix. Below we will show that $\mathbf{G}\left(\sigma_{j}\right) \in \Re^{N^{2} \times N^{2}}$ is a P-matrix. From Lemma 2, $\mathbf{G}\left(\sigma_{j}\right)$ is a P-matrix if and only if, for any $x \in \Re^{N^{2}}$,

$$
x_{i}\left(\mathbf{G}\left(\sigma_{j}\right) x\right)_{i} \leq 0, \quad \forall i \Rightarrow x=0 .
$$

Let us denote

$$
x=\left(\begin{array}{c}
x^{1} \\
x^{2} \\
\vdots \\
x^{N}
\end{array}\right),
$$

where $x^{p} \in \Re^{N}, p=1,2, \cdots, N$. Then, we can write

$$
\mathbf{G}\left(\sigma_{j}\right) x=\left(\begin{array}{c}
\hat{\mathbf{M}}\left(\sigma_{j}\right) x^{1}+\hat{\mathbf{M}}^{\prime}\left(\sigma_{j}\right) x^{2} \\
\hat{\mathbf{M}}\left(\sigma_{j}\right) x^{2}+\hat{\mathbf{M}}^{\prime}\left(\sigma_{j}\right) x^{3} \\
\vdots \\
\hat{\mathbf{M}}\left(\sigma_{j}\right) x^{N-1}+\hat{\mathbf{M}}^{\prime}\left(\sigma_{j}\right) x^{N} \\
\hat{\mathbf{M}}\left(\sigma_{j}\right) x^{N}
\end{array}\right) .
$$

Assume

$$
x_{i}\left(\mathbf{G}\left(\sigma_{j}\right) x\right)_{i} \leq 0, \quad \forall i .
$$

First, we show $x^{N}=0$. By Lemma 2 , since $\hat{\mathbf{M}}\left(\sigma_{j}\right)$ is a P-matrix, we have

$$
y_{i}\left(\hat{\mathbf{M}}\left(\sigma_{j}\right) y\right)_{i} \leq 0, \quad \forall i \Rightarrow y=0
$$

for any $y \in \Re^{N}$. It then follows from (5.8), (5.9) and (5.10) that $x^{N}=0$.

Next, notice that the $(N-1)$ th block of the vector $\mathbf{G}\left(\sigma_{j}\right) x$ equals $\hat{\mathbf{M}}\left(\sigma_{j}\right) x^{N-1}$ since $x^{N}=0$. Hence, by the same reasoning as above, we have $x^{N-1}=0$. Repeating similar arguments, we deduce $x^{N-2}=x^{N-3}=\cdots=x^{1}=0$, implying (5.7) hold. Thus, $\mathbf{G}\left(\sigma_{j}\right)$ is a P-matrix. Since every P-matrix is an $R_{0}$ matrix [9], it follows from Lemma 3 that the solution set of the optimization problem (4.7) is nonempty and bounded.

From Proposition 1 and Proposition 2, if we choose the step-size parameter $\delta t$ small enough to satisfy the conditions (5.1) and (5.6), respectively, then we can ensure that the linear complementarity problem (4.3) in the expected value method and the optimization problem (4.7) in the expected residual minimization method have a solution. However, when $\delta t$ is small, the size of problem (4.3) or (4.7) becomes large, which may make the problem more expensive computationally. 


\section{$6 \quad$ Numerical experiments}

In this section, we describe numerical experiments. All computations were carried out using Matlab on a PC. We use put options whose underlying asset is S\&P100. S\&P100 is a market value weighted index consisting of 100 leading United States stocks. First, we state how to set the parameters of the stochastic linear complementarity problem (4.1). Next, we describe some criteria used to compare the results. Finally, we show and discuss the computational results.

\subsection{Parameter setting}

In this subsection, we describe how to set the parameters to derive the stochastic complementarity problem (4.1).

We set the parameters in the finite difference approximation as $\theta_{1}=1 / 2$ and $\theta_{2}=1 / 2$. We set $L=4$ to divide the time interval [0,T], where $T$ is the expiration date. From (2.9), the length of each subinterval is $\delta t=T / 4$. We assume that the underlying asset does not exceed a large positive number $S_{\max }=900$. Then the interval $\left[0, S_{\max }\right]$ is divided into $N$ subintervals of equal length, where we set $N=30$. From (2.10), the length of each subinterval is $\delta S=S_{\max } / N=30$. The payoff $\Lambda(S, t)$ is discretized for the asset price and the time. Since we consider put options, the elements of $\boldsymbol{\Lambda}^{l}, l=0,1, \cdots, L$ can be written as

$$
\Lambda_{n}^{l}= \begin{cases}E-n \delta S & 1 \leq n \leq E / \delta S \\ 0 & E / \delta S<n \leq N \quad ; \quad 0 \leq l \leq L .\end{cases}
$$

We obtained the data listed in Appendix from the Wall Street Journal's homepage ${ }^{1}$. Here, we use the interest rate of 6 month U.S. government bond obtained from the same page as the risk-free rate $r$. From the data, we give the risk-free rate $r$ and, noting that the expiration date is the third Friday of the expiration month, calculate the expiration date $T$. Then, using the strike price $E$, we calculate the payoff $\boldsymbol{\Lambda}^{l}, l=0,1, \cdots, L$ from (6.1).

For example, for the option whose expiration month is January, 2010 and whose strike price is 360 on November 30, 2009, the risk-free rate is $r=0.00242$ and the expiration date is $T=46 / 365$, since there are 46 days from November 30 to January 15 (the third Friday of January, 2010). Since the strike price is $E=360$ and we set $\delta S=30, N=30$ and $L=4$, we have $E / \delta S=360 / 30=12$ and, from (6.1), we have

$$
\Lambda_{n}^{l}= \begin{cases}360-30 n & 1 \leq n \leq 12 \\ 0 & 12<n \leq 30 \quad ; \quad 0 \leq l \leq 4 .\end{cases}
$$

Now we describe how to estimate the volatility of the rate of return of S\&P100. From the Yahoo! finance homepage ${ }^{2}$, we obtain the historical data $S_{0}, \cdots, S_{180}$, where $S_{t}$ is the asset price observed $t$ days ago. From the historical data, we calculate the continuously compounded rates of return of $\mathrm{S} \& \mathrm{P} 100$ as

$$
u_{t}=\ln \left(\frac{S_{t-1}}{S_{t}}\right), \quad t=1, \cdots, 180 .
$$

Using the continuously compounded rates of return $u_{1}, \cdots, u_{180}$, we obtain the average of the continuously compounded rate of return in the most recent 60 days, the average rate of the

\footnotetext{
${ }^{1}$ http://asia.wsj.com/home-page

${ }^{2}$ http://finance.yahoo.com
} 
return in the next 60 days, and the average rate of the return in the remaining 60 days, denoted as $\bar{u}^{1}, \bar{u}^{2}$ and $\bar{u}^{3}$, respectively, by the following formulas:

$$
\bar{u}^{1}=\frac{1}{60} \sum_{t=1}^{60} u_{t}, \quad \bar{u}^{2}=\frac{1}{60} \sum_{t=61}^{120} u_{t}, \quad \bar{u}^{3}=\frac{1}{60} \sum_{t=121}^{180} u_{t} .
$$

Similarly, we compute the volatilities of the continuously compounded rate of return in the above-mentioned three periods by

$$
\sigma_{1}=\sqrt{\frac{250}{59} \sum_{t=1}^{60}\left(u_{t}-\bar{u}^{1}\right)^{2}}, \sigma_{2}=\sqrt{\frac{250}{59} \sum_{t=61}^{120}\left(u_{t}-\bar{u}^{2}\right)^{2}}, \sigma_{3}=\sqrt{\frac{250}{59} \sum_{t=121}^{180}\left(u_{t}-\bar{u}^{3}\right)^{2}} .
$$

We regard these values as realizations of the volatility and set $P\left\{\sigma=\sigma_{j}\right\}=1 / 3, j=1,2,3$. Since there are 250 business days in a year, the right-hand side of (6.2) contains the factor $\sqrt{250}$ to convert the day rate of the volatility into the annual rate of the volatility. Since we adopt the unbiased variance, the right-hand side of (6.2) contains the factor $1 / \sqrt{59}$ rather than $1 / \sqrt{60}$.

We use these parameter values to calculate the coefficients in the stochastic linear complementarity problem (4.1). Then, we obtain the deterministic formulations based on the expected value method (4.3) and the expected residual minimization method (4.7). We let $\mathbf{V}_{\mathrm{EV}}$ and $\mathbf{V}_{\text {ERM }}$ denote the solutions obtained by the expected value method and the expected residual minimization method, respectively. In the expected residual minimization method, we adopt the min function (3.4) and the FB function (3.5) as an NCP function and set the parameter $\nu$ in (3.4) and (3.5) as $\nu=0.1,1,10$. We use the PATH solver [11] to solve the linear complementarity problem (4.3) and use the fmincon solver in the Matlab Toolbox to solve the optimization problem (4.7).

\subsection{Criteria for comparing solutions}

In this subsection, we describe two criteria used to compare solutions obtained by different formulations. As a standard for comparison, we use the simple average of the solutions $\mathbf{V}_{j}$ obtained by solving the linear complementarity problems (4.1) for $\sigma_{j}, j=1, \cdots, k$ :

$$
\mathbf{V}_{\mathrm{avg}}=\frac{1}{k} \sum_{j=1}^{k} \mathbf{V}_{j}
$$

\subsubsection{Estimation error}

One criterion is to analyze the prices obtained from each method against the prices observed in the real market.

First, we describe how the prices of options corresponding to the current asset price can be estimated from the solutions $\mathbf{V}_{\mathrm{EV}}, \mathbf{V}_{\mathrm{ERM}}$ and $\mathbf{V}_{\text {avg }}$. Note that the vector $\mathbf{V}$ can be written as

$$
\mathbf{V}=\left(\begin{array}{c}
\mathbf{V}^{0} \\
\mathbf{V}^{1} \\
\vdots \\
\mathbf{V}^{l} \\
\vdots \\
\mathbf{V}^{L-2} \\
\mathbf{V}^{L-1}
\end{array}\right) \text { with } \mathbf{V}^{l}=\left(\begin{array}{c}
V_{1}^{l} \\
\vdots \\
V_{n}^{l} \\
\vdots \\
V_{N}^{l}
\end{array}\right), \quad l=0,1, \cdots, L-1
$$


From $(2.11), V_{n}^{l}$ is the price corresponding to time $t_{l}$ and asset price $S_{n}$, where $t_{l}$ and $S_{n}$ are given by (2.9) and (2.10), respectively. Recall that we want to obtain the prices of options corresponding to the asset price "at present", i.e., $t=0$. We can get the prices of options at $t=0$ by taking the first block component of the solution $\mathbf{V}$, i.e., $\mathbf{V}^{0}$.

Notice that the solution $\mathbf{V}$ gives us only the prices corresponding to $N$ asset prices $S_{n}, n=$ $1, \cdots, N$. We want to obtain the prices of options corresponding to the given asset price at $t=0$. This can be obtained by the following procedure: Suppose that the given asset price lies in the interval $[n \delta S,(n+1) \delta S]$ for some integer $n>0$, i.e., it is represented as $n \delta S+a$, where $a$ is a positive number such that $0 \leq a<\delta S$. Then we can obtain the corresponding price of options by interpolating the value of the $n$th element of $\mathbf{V}^{0}$ and the $(n+1)$ th element of $\mathbf{V}^{0}$. Specifically, we calculate the prices of options by the formula:

$$
\left(1-\frac{a}{\delta S}\right) V_{n}^{0}+\frac{a}{\delta S} V_{n+1}^{0}
$$

For example, look at the data on November 30, 2009. The current asset price is $\$ 511$. If we set $\delta S=30$, then 511 is in between $510(=17 \delta S)$ and $540(=18 \delta S)$. So we use the values of $V_{17}^{0}$ and $V_{18}^{0}$ in the solution V. Since we can write $511=17 \delta S+1$, we obtain the corresponding price of options by computing

$$
\left(1-\frac{1}{30}\right) V_{17}^{0}+\frac{1}{30} V_{18}^{0}
$$

Let $Z$ be the number of options used for numerical experiments. Then, for the $z$ th option $(z=1,2, \cdots, Z)$, its prices corresponding to the given asset price at $t=0$ obtained by the expected value method, the expected residual minimization method, and the simple average method as $V_{\mathrm{EV}}^{z}, V_{\mathrm{ERM}}^{z}$ and $V_{\text {avg }}^{z}$, respectively. We get the prices of options observed in the real market from the data listed in the appendix and denote it as $V_{\mathrm{mkt}}^{z}$. For example, the price of the option whose expiration date is January, 2010 and strike price is $\$ 360$ on November 30, 2009 is $\$ 0.35$.

Using these values, we calculate the root mean squared error rate (RMSER)

$$
\operatorname{RMSER}_{i}=\sqrt{\frac{1}{Z} \sum_{z=1}^{Z}\left(\frac{V_{i}^{z}-V_{\mathrm{mkt}}^{z}}{V_{\mathrm{mkt}}^{z}}\right)^{2}}, \quad i=\mathrm{avg}, \mathrm{EV}, \mathrm{ERM}
$$

in order to compare the preciseness of estimation. We also calculate the mean error rate (MER)

$$
\mathrm{MER}_{i}=\frac{1}{Z} \sum_{z=1}^{Z}\left(\frac{V_{i}^{z}-V_{\mathrm{mkt}}^{z}}{V_{\mathrm{mkt}}^{z}}\right), \quad i=\mathrm{avg}, \mathrm{EV}, \mathrm{ERM}
$$

in order to compare the bias of the price obtained by each method relative to the price observed in the real market.

\subsubsection{Measures of feasibility and optimality}

Another criterion is to compare the solutions obtained by each method in terms of some measures of feasibility and optimality, as was done in [7]. For any $\omega \in \Omega$, the complementarity problem

$$
0 \leq x \perp F(x, \omega) \geq 0
$$


is equivalent to the optimization problem

$$
\begin{aligned}
\min & x^{T} F(x, \omega) \\
\text { s.t. } & x \geq 0, \quad F(x, \omega) \geq 0
\end{aligned}
$$

in the sense that an optimal solution of (6.5) with zero objective value coincides with a solution of (6.4). We denote the discrete samples of $\omega$ as $\left\{\omega_{j}, j=1, \cdots, k\right\}$ and denote the solution obtained by the expected value method (3.3) or the expected residual minimization method (3.8) as $x^{*}$.

Using the ideas from the literature on stochastic programming $[2,17,18]$, we evaluate the violation of the inequality condition $F(x, \omega) \geq 0$ in problem (6.5) by

$$
\gamma_{\mathrm{feas}}\left(x^{*}, \omega\right)=\left\|\min \left(0, F\left(x^{*}, \omega\right)\right)\right\|,
$$

and evaluate the loss in the objective function of (6.5) by

$$
\gamma_{\mathrm{opt}}\left(x^{*}, \omega\right)=x^{* T} \max \left(0, F\left(x^{*}, \omega\right)\right) .
$$

Here $\min \left(0, F\left(x^{*}, \omega\right)\right)$ and $\max \left(0, F\left(x^{*}, \omega\right)\right)$ denote the vectors with components $\min \left(0, F_{i}\left(x^{*}, \omega\right)\right)$ and $\max \left(0, F_{i}\left(x^{*}, \omega\right)\right)$, respectively, where $F_{i}\left(x^{*}, \omega\right)$ is the $i$ th element of $F\left(x^{*}, \omega\right)$. We apply these measures to the stochastic linear complementarity problem (4.4) derived from the model for pricing American options with uncertain volatility. As in Subsection 6.1, we denote the solution from the expected value method (4.3), the expected residual minimization method, and the simple average method as $\mathbf{V}_{\mathrm{EV}}, \mathbf{V}_{\mathrm{ERM}}$ and $\mathbf{V}_{\mathrm{avg}}$, respectively. Then, (6.6) and (6.7) are written as

$$
\begin{gathered}
\gamma_{\mathrm{feas}}\left(\mathbf{V}_{i}, \sigma\right)=\sqrt{\sum_{l=0}^{L-1}\left\|\min \left(0, \mathbf{M}(\sigma) \mathbf{V}_{i}^{l}+\mathbf{M}^{\prime}(\sigma) \mathbf{V}_{i}^{l+1}\right)\right\|^{2}}, \quad i=\operatorname{avg}, \text { EV , ERM, } \\
\gamma_{\mathrm{opt}}\left(\mathbf{V}_{i}, \sigma\right)=\sum_{l=0}^{L-1}\left(\mathbf{V}_{i}^{l}-\boldsymbol{\Lambda}^{l}\right)^{T} \max \left(0, \mathbf{M}(\sigma) \mathbf{V}_{i}^{l}+\mathbf{M}^{\prime}(\sigma) \mathbf{V}_{i}^{l+1}\right), \quad i=\operatorname{avg}, \text { EV }, \text { ERM, }
\end{gathered}
$$

respectively. We denote the discrete samples of $\sigma$ as $\left\{\sigma_{j}, j=1, \cdots, k\right\}$. For the $z$ th option and $\sigma=\sigma_{j}$, we denote the values of $\gamma_{\text {feas }}\left(\mathbf{V}_{i}, \sigma\right)$ and $\gamma_{\text {opt }}\left(\mathbf{V}_{i}, \sigma\right)$ as $\gamma_{\text {feas }}\left(\mathbf{V}_{i}^{z}, \sigma_{j}\right)$ and $\gamma_{\text {opt }}\left(\mathbf{V}_{i}^{z}, \sigma_{j}\right)$, respectively. We calculate the average values of $\gamma_{\text {feas }}\left(\mathbf{V}_{i}^{z}, \sigma_{j}\right)$ and $\gamma_{\mathrm{opt}}\left(\mathbf{V}_{i}^{z}, \sigma_{j}\right)$ for $\sigma_{j}, j=1, \cdots, k$ and $z=1, \cdots, Z$ by the following formulas:

$$
\begin{aligned}
\Gamma_{i}^{\text {feas }} & =\frac{1}{k Z} \sum_{z=1}^{Z} \sum_{j=1}^{k} \gamma_{\text {feas }}\left(\mathbf{V}_{i}^{z}, \sigma_{j}\right), \quad i=\text { avg, EV }, \text { ERM }, \\
\Gamma_{i}^{\mathrm{opt}} & =\frac{1}{k Z} \sum_{z=1}^{Z} \sum_{j=1}^{k} \gamma_{\mathrm{opt}}\left(\mathbf{V}_{i}^{z}, \sigma_{j}\right), \quad i=\mathrm{avg}, \mathrm{EV}, \mathrm{ERM},
\end{aligned}
$$

where $Z$ is the total number of options used for numerical experiments. We use $\Gamma_{i}^{\text {feas }}$ and $\Gamma_{i}^{\text {opt }}$ to compare solutions obtained by the different methods. 


\begin{tabular}{|c|c|}
\hline moneyness & category \\
\hline \hline $1.2<S / E$ & deep-out-of-the-money(DOTM) \\
\hline $1.04<S / E \leq 1.2$ & out-of-the-money(OTM) \\
\hline $0.98 \leq S / E \leq 1.04$ & at-the-money(ATM) \\
\hline$S / E<0.98$ & in-the-money(ITM) \\
\hline
\end{tabular}

Table 1: The classification according to moneyness

\subsection{Numerical results}

In this subsection, we show the numerical results. First, we show the values of RMSER and MER in Table 2 and Table 3, respectively. In both tables, we classify options into 4 categories according to their moneyness [1] which is the asset price $S$ divided by the strike price $E$, as shown in Tables 1. The numbers of options used in the numerical experiments are 88 for DOTM, 148 for OTM, 66 for ATM, and 47 for ITM.

In terms of RMSER, the most precise estimate of the prices of options observed in the real market is given by the expected residual minimization method using the FB function (3.5) with parameter $\nu=1$. If we focus on ATM and ITM, the expected residual minimization method using the FB function (3.5) with parameter $\nu=0.1$ estimates most precisely the prices of options observed in the real market. Regarding the positive parameter $\nu$, the best choice to estimate the prices observed in the real market is $\nu=1$ for both the min function and the FB function. If we set $\nu=10$, the values of RMSER become large, that is to say, the method fails to estimate the prices observed in the real market. So we may conclude that $\nu=1$ or even a smaller value is an appropriate choice. In terms of MER, all the prices $V_{\mathrm{avg}}, V_{\mathrm{EV}}$ and $V_{\mathrm{ERM}}$, except those obtained by the expected residual minimization method using the min function with parameter $\nu=10$ and the FB function with parameter $\nu=10$, tend to be much lower than the prices observed in the real market.

We show the values of $\Gamma^{\text {feas }}$ defined in Subsection 6.2.2 in Table 4. Table 5 shows the values $\Gamma^{\mathrm{opt}}$ divided by 100 . The solution $\mathbf{V}_{\mathrm{ERM}}$ by the expected residual minimization method has smaller $\Gamma^{\text {feas }}$ values and larger $\Gamma^{\mathrm{opt}}$ values than the other solutions. Recall that the inequality (2.5) is derived from the no arbitrage assumption and $\Gamma^{\text {feas }}$ represents the violation of this inequality. Thus, the expected residual minimization method (4.7) produces a solution which tends to satisfy no arbitrage assumption, which is one of the most important assumptions in the theory of options. Regarding the positive parameter $\nu$ in (3.4) and (3.5), the larger $\nu$ we set, the smaller $\Gamma^{\text {feas }}$ values the solution has, that is to say, the stronger tendency to satisfy the no arbitrage assumption the solution has. However, the formulation with a large $\nu$ may yield a solution with a large $\Gamma^{\mathrm{opt}}$ value. 


\begin{tabular}{|c|c|c|c|c|c|c|c|c|}
\cline { 4 - 9 } \multicolumn{1}{c|}{} & \multirow{2}{*}{ avg } & \multirow{2}{*}{$\mathrm{EV}$} & \multicolumn{9}{|c|}{ ERM } \\
\cline { 4 - 9 } \multicolumn{1}{c|}{} & & & \multicolumn{3}{|c|}{$\min$} & \multicolumn{3}{c|}{ FB } \\
\cline { 4 - 9 } & & & $\nu=0.1$ & $\nu=1$ & $\nu=10$ & $\nu=0.1$ & $\nu=1$ & $\nu=10$ \\
\hline DOTM & 0.95 & 0.97 & 0.97 & 0.84 & 13.25 & 0.94 & 0.78 & 18.99 \\
\hline OTM & 0.65 & 0.67 & 0.65 & 0.47 & 2.54 & 0.56 & 0.46 & 8.87 \\
\hline ATM & 0.18 & 0.17 & 0.16 & 0.22 & 0.34 & 0.16 & 0.21 & 0.40 \\
\hline ITM & 0.07 & 0.07 & 0.07 & 0.08 & 0.11 & 0.06 & 0.08 & 0.38 \\
\hline total & 0.64 & 0.66 & 0.65 & 0.53 & 6.86 & 0.60 & 0.50 & 11.15 \\
\hline
\end{tabular}

Table 2: Comparison of RMSER

\begin{tabular}{|c|c|c|c|c|c|c|c|c|}
\cline { 4 - 9 } \multicolumn{1}{c|}{} & \multirow{2}{*}{\multicolumn{1}{c|}{ avg }} & \multirow{2}{*}{ EV } & \multicolumn{9}{|c|}{ ERM } \\
\cline { 4 - 9 } \multicolumn{1}{c|}{} & & & \multicolumn{3}{|c|}{$\min$} & \multicolumn{3}{c|}{ FB } \\
\cline { 4 - 9 } & & & $\nu=0.1$ & $\nu=1$ & $\nu=10$ & $\nu=0.1$ & $\nu=1$ & $\nu=10$ \\
\hline DOTM & -0.95 & -0.97 & -0.97 & -0.82 & 3.48 & -0.94 & -0.76 & 6.17 \\
\hline OTM & -0.67 & -0.68 & -0.66 & -0.41 & 0.51 & -0.55 & -0.41 & 1.40 \\
\hline ATM & -0.18 & -0.17 & -0.13 & 0.08 & 0.15 & -0.04 & 0.07 & 0.21 \\
\hline ITM & -0.04 & -0.04 & -0.03 & 0.03 & 0.04 & -0.01 & 0.02 & 0.10 \\
\hline total & -0.56 & -0.57 & -0.56 & -0.36 & 1.13 & -0.48 & -0.35 & 2.20 \\
\hline
\end{tabular}

Table 3: Comparison of MER

\begin{tabular}{|c|c|c|c|c|c|c|c|c|}
\cline { 4 - 9 } \multicolumn{1}{c|}{} & \multirow{2}{*}{} & \multirow{2}{*}{ avg } & \multirow{2}{*}{ EV } & \multicolumn{6}{|c|}{ ERM } \\
\cline { 4 - 9 } \multicolumn{1}{c|}{} & & & $\nu=0.1$ & $\nu=1$ & $\nu=10$ & $\nu=0.1$ & $\nu=1$ & $\nu=10$ \\
\hline DOTM & 24.15 & 22.63 & 19.99 & 2.17 & 0.11 & 11.22 & 1.00 & 0.07 \\
\hline OTM & 27.63 & 25.94 & 22.32 & 2.54 & 0.11 & 12.42 & 1.09 & 0.08 \\
\hline ATM & 32.40 & 30.31 & 25.71 & 2.49 & 0.08 & 13.98 & 1.16 & 0.07 \\
\hline ITM & 31.66 & 29.68 & 25.65 & 3.43 & 0.06 & 14.55 & 1.31 & 0.07 \\
\hline total & 28.20 & 26.44 & 22.82 & 2.56 & 0.10 & 12.70 & 1.11 & 0.07 \\
\hline
\end{tabular}

Table 4: Comparison of $\Gamma^{\text {feas }}$

\begin{tabular}{|c|c|c|c|c|c|c|c|c|}
\cline { 4 - 9 } \multicolumn{1}{c|}{} & \multirow{2}{*}{\multicolumn{2}{c|}{ avg }} & \multirow{2}{*}{$\mathrm{EV}$} & \multicolumn{6}{|c|}{ ERM } \\
\cline { 4 - 9 } \multicolumn{1}{c|}{} & & & \multicolumn{3}{|c|}{$\min$} & \multicolumn{3}{c|}{ FB } \\
\cline { 4 - 9 } & & $\nu=0.1$ & $\nu=1$ & $\nu=10$ & $\nu=0.1$ & $\nu=1$ & $\nu=10$ \\
\hline DOTM & 2.39 & 2.85 & 3.45 & 11.50 & 267.84 & 5.84 & 10.95 & 170.07 \\
\hline OTM & 3.07 & 3.63 & 4.47 & 14.76 & 344.31 & 7.45 & 13.00 & 392.87 \\
\hline ATM & 3.86 & 4.57 & 5.76 & 18.29 & 116.77 & 9.61 & 16.28 & 111.62 \\
\hline ITM & 4.26 & 5.02 & 6.17 & 21.92 & 49.42 & 10.10 & 18.79 & 346.91 \\
\hline total & 3.21 & 3.80 & 4.69 & 15.57 & 242.29 & 7.81 & 13.88 & 277.31 \\
\hline
\end{tabular}

Table 5: Comparison of $\Gamma^{\text {opt }}$ 


\section{Conclusion}

In this paper, we have proposed two deterministic formulations for pricing American options with uncertain volatility based on the expected value method and the expected residual minimization method for stochastic linear complementarity problems. We have shown sufficient conditions that guarantee the formulations by the expected value method and the expected residual minimization method to have solutions. The numerical results indicate that the expected residual minimization method yield solutions that tend to satisfy the no arbitrage assumption than the expected value method.

\section{References}

[1] Bakshi, G., Cao, C., Chen, Z.: Empirical performance of alternative option pricing models. The Journal of Finance. 52, 2003-2049 (1997)

[2] Birge, J. R., Louveaux, F.: Introduction to Stochastic Programming. Springer-Verlag, New York (1997)

[3] Black, F., Sholes, M.: The pricing of options and corporate liabilities. Journal of Political Economy. 4, 637-654 (1973)

[4] Boyle, P., Phelim, P.: Options: A Monte Carlo approach. Journal of Finance Economics. 4, 323-338 (1977)

[5] Brennan, M. J., Schwartz, E. S.: The valuation of American put option. The Journal of Finance. 32, 449-462 (1977)

[6] Chen, X., Fukushima, M.: Expected residual minimization method for stochastic linear complementarity problems. Mathematics of Operations Research. 30, 1022-1038 (2004)

[7] Chen, X., Zhang, C., Fukushima, M.: Robust solution of monotone stochastic linear complementarity problems. Mathematical Programming. 117, 51-80 (2009)

[8] Clark, N., Parrott, K.: Multigrid for American option pricing with stochastic volatility. Applied Mathematical Finance. 6, 177-195 (1999)

[9] Cottle, R. W., Pang, J. S., Stone, R. E.: The Linear Complementarity Problem. Academic Press, San Diego (1992)

[10] Cox, J. C., Ross, S. A., Rubinstein, M.: Option pricing: A simplified approach. Journal of Finance Economics. 7, 229-268 (1979)

[11] Dirkse, S. P., Ferris, M. C.: The PATH solver: A non-monotone stabilization for mixed complementarity problems. Optimization Methods and Software. 5, 123-156 (1995)

[12] Facchinei, F., Pang, J. S.: Finite-Dimensional Variational Inequalities and Complementarity Problems, I and II. Springer-Verlag, New York (2003)

[13] Gurkan, G., Ozge, A. Y., Robinson, S. M.: Sample-path solution of stochastic variational inequalities. Mathematical Programming. 84, 313-333 (1999) 
[14] Heston, S. L.: A closed form solution for options with stochastic volatility with applications to bond and currency options. Reviews of Financial Studies. 6, 327-343 (1993)

[15] Huang, J., Pang, J. S.: Option pricing and linear complementarity. The Journal of Computational Finance. 2, 31-60 (1998)

[16] Hull, J. C.: Options, Futures, and Other Derivatives. Prentice Hall, Upper Saddle River (2000)

[17] Kall, P., Wallace, S. W.: Stochastic Programming. John Wiley \& Sons (1994)

[18] Marti, K.: Stochastic Optimization Methods. Springer-Verlag, Berlin (2005)

[19] Svensson, O., Vorobyov, S.: A subexponential algorithm for a subclass of P-matrix generalized linear complementarity problems. Technical Report, DIMACS (2005)

[20] Telly, J. A.: Valuing American options in path simulation model. Transactions of Society of Actuaries. XLV, 499-520 (1994)

[21] Wilmott, P., Dewynne, J. N., Howison, S.: The Mathematics of Financial Derivatives - A Student Introduction. Cambridge University Press, Cambridge (1995)

[22] Zvan, R., Forsyth, P. A., Vetzal, K. R.: Penalty methods for American options with stochastic volatility. Journal of Computational and Applied Mathematics. 91, 119-218 (1998) 


\section{A Data for numerical experiments}

Nov 30, 2009 Asset price : 511

Risk-free rate r: $0.152 \%$

\begin{tabular}{|c|c|c|}
\hline Expiration & Strike & Price \\
\hline Jan & 360 & 0.35 \\
\hline Jan & 390 & 0.55 \\
\hline Jan & 420 & 1.33 \\
\hline Jan & 450 & 2.8 \\
\hline Jan & 510 & 17.1 \\
\hline Jan & 540 & 38.2 \\
\hline Feb & 360 & 0.9 \\
\hline Feb & 450 & 6.4 \\
\hline Mar & 420 & 5.6 \\
\hline Mar & 480 & 15.28 \\
\hline
\end{tabular}

Dec 15, 2009 Asset price : 512

Risk-free rate r : $0.164 \%$

\begin{tabular}{|c|c|c|}
\hline Expiration & Strike & Price \\
\hline Feb & 300 & 0.05 \\
\hline Feb & 390 & 0.9 \\
\hline Feb & 420 & 1.75 \\
\hline Feb & 510 & 16.45 \\
\hline Mar & 360 & 0.85 \\
\hline Mar & 420 & 3.3 \\
\hline Mar & 480 & 11.5 \\
\hline
\end{tabular}

Dec 21, 2009 Asset price : 514

Risk-free rate r : $0.165 \%$

\begin{tabular}{|c|c|c|}
\hline Expiration & Strike & Price \\
\hline Jan & 450 & 0.6 \\
\hline Feb & 450 & 2.8 \\
\hline Feb & 480 & 6.1 \\
\hline Feb & 510 & 14.39 \\
\hline Mar & 480 & 10 \\
\hline Mar & 510 & 18.7 \\
\hline Mar & 540 & 35.9 \\
\hline
\end{tabular}

Dec 8, 2009 Asset price : 507

Risk-free rate r : $0.147 \%$

\begin{tabular}{|c|c|c|}
\hline Expiration & Strike & Price \\
\hline Jan & 390 & 0.4 \\
\hline Jan & 420 & 0.9 \\
\hline Jan & 450 & 2.06 \\
\hline Jan & 480 & 5.5 \\
\hline Feb & 300 & 0.15 \\
\hline Feb & 390 & 1.5 \\
\hline Feb & 420 & 2.85 \\
\hline Feb & 450 & 5.5 \\
\hline Feb & 480 & 10.9 \\
\hline Feb & 510 & 21.64 \\
\hline Mar & 360 & 1.45 \\
\hline Mar & 480 & 15.1 \\
\hline
\end{tabular}

Dec 16, 2009 Asset price : 506 Risk-free rate r : $0.152 \%$

\begin{tabular}{|c|c|c|}
\hline Expiration & Strike & Price \\
\hline Jan & 420 & 0.55 \\
\hline Jan & 450 & 1.4 \\
\hline Jan & 480 & 3.8 \\
\hline Jan & 510 & 12.2 \\
\hline Feb & 420 & 1.75 \\
\hline Feb & 450 & 4.11 \\
\hline Feb & 480 & 9 \\
\hline Feb & 510 & 19.52 \\
\hline Mar & 420 & 3.8 \\
\hline
\end{tabular}

Dec 22, 2009 Asset price : 515 Risk-free rate r : $0.172 \%$

\begin{tabular}{|c|c|c|}
\hline Expiration & Strike & Price \\
\hline Jan & 480 & 1.4 \\
\hline Jan & 510 & 6.5 \\
\hline Jan & 420 & 1.05 \\
\hline Feb & 480 & 5.8 \\
\hline Feb & 510 & 13.3 \\
\hline Mar & 480 & 9 \\
\hline Mar & 510 & 18.4 \\
\hline
\end{tabular}

Dec 11, 2009 Asset price : 514 Risk-free rate r : $0.157 \%$

\begin{tabular}{|c|c|c|}
\hline Expiration & Strike & Price \\
\hline Jan & 420 & 0.6 \\
\hline Jan & 450 & 1.31 \\
\hline Jan & 480 & 3.26 \\
\hline Feb & 480 & 8.4 \\
\hline Mar & 420 & 3.73 \\
\hline Mar & 480 & 12.33 \\
\hline
\end{tabular}

Dec 18, 2009 Asset price : 509

Risk-free rate r : $0.152 \%$

\begin{tabular}{|c|c|c|}
\hline Expiration & Strike & Price \\
\hline Jan & 360 & 0.1 \\
\hline Jan & 420 & 0.53 \\
\hline Jan & 450 & 1.2 \\
\hline Jan & 510 & 11 \\
\hline Feb & 480 & 8.5 \\
\hline Mar & 420 & 3.7 \\
\hline Mar & 540 & 41.8 \\
\hline
\end{tabular}

Dec 23, 2009 Asset price : 515

Risk-free rate r : $0.172 \%$

\begin{tabular}{|c|c|c|}
\hline Expiration & Strike & Price \\
\hline Jan & 390 & 0.1 \\
\hline Jan & 450 & 0.5 \\
\hline Jan & 510 & 6 \\
\hline Feb & 390 & 0.45 \\
\hline Feb & 450 & 2.25 \\
\hline Feb & 480 & 5.4 \\
\hline Feb & 510 & 13.17 \\
\hline Mar & 420 & 2.25 \\
\hline Mar & 540 & 34.9 \\
\hline
\end{tabular}


Dec 24, 2009 Asset price : 518

Risk-free rate r : $0.168 \%$

\begin{tabular}{|c|c|c|}
\hline Expiration & Strike & Price \\
\hline Jan & 420 & 0.2 \\
\hline Jan & 450 & 0.45 \\
\hline Jan & 480 & 1.14 \\
\hline Jan & 510 & 5 \\
\hline Feb & 420 & 0.89 \\
\hline Feb & 480 & 4.8 \\
\hline Mar & 420 & 2 \\
\hline Mar & 450 & 4 \\
\hline Mar & 540 & 32.9 \\
\hline
\end{tabular}

Risk-free rate r : $0.157 \%$

\begin{tabular}{|c|c|c|}
\hline Expiration & Strike & Price \\
\hline Feb & 390 & 0.25 \\
\hline Feb & 450 & 0.95 \\
\hline Feb & 480 & 2.47 \\
\hline Feb & 510 & 7 \\
\hline Mar & 420 & 1.2 \\
\hline Mar & 450 & 2.7 \\
\hline Mar & 480 & 5.6 \\
\hline Apr & 420 & 2.45 \\
\hline Apr & 480 & 8.5 \\
\hline
\end{tabular}

Jan 11, 2010 Asset price : 529

Risk-free rate r : $0.13 \%$

\begin{tabular}{|c|c|c|}
\hline Expiration & Strike & Price \\
\hline Feb & 420 & 0.31 \\
\hline Feb & 450 & 0.65 \\
\hline Feb & 480 & 1.55 \\
\hline Feb & 510 & 5.45 \\
\hline Feb & 570 & 43.8 \\
\hline Mar & 360 & 0.15 \\
\hline Mar & 420 & 0.9 \\
\hline Mar & 450 & 1.8 \\
\hline Mar & 480 & 4.2 \\
\hline Mar & 510 & 9.82 \\
\hline Mar & 570 & 43.8 \\
\hline Apr & 360 & 0.5 \\
\hline
\end{tabular}

Jan 4, 2010 Asset price : 523

Risk-free rate r : $0.172 \%$

\begin{tabular}{|c|c|c|}
\hline Expiration & Strike & Price \\
\hline Feb & 420 & 0.7 \\
\hline Feb & 450 & 1.34 \\
\hline Feb & 480 & 3.25 \\
\hline Feb & 510 & 8.8 \\
\hline Feb & 570 & 50 \\
\hline Mar & 420 & 1.55 \\
\hline Mar & 480 & 6.5 \\
\hline Mar & 510 & 13.6 \\
\hline Mar & 540 & 28.6 \\
\hline Apr & 480 & 9.53 \\
\hline
\end{tabular}

Jan 7, 2010 Asset price : 526

Risk-free rate r : $0.162 \%$

\begin{tabular}{|c|c|c|}
\hline Expiration & Strike & Price \\
\hline Feb & 390 & 0.2 \\
\hline Feb & 480 & 2.18 \\
\hline Feb & 510 & 6.5 \\
\hline Mar & 390 & 0.6 \\
\hline Mar & 420 & 1.2 \\
\hline Apr & 420 & 2.1 \\
\hline
\end{tabular}

Jan 12, 2010 Asset price : 524

Risk-free rate r : $0.137 \%$

\begin{tabular}{|c|c|c|}
\hline Expiration & Strike & Price \\
\hline Feb & 420 & 0.35 \\
\hline Feb & 450 & 0.9 \\
\hline Feb & 480 & 2.2 \\
\hline Feb & 510 & 7.2 \\
\hline Mar & 480 & 5.4 \\
\hline Mar & 510 & 11.68 \\
\hline
\end{tabular}

Jan 5, 2010 Asset price : 525

Risk-free rate r : $0.165 \%$

\begin{tabular}{|c|c|c|}
\hline Expiration & Strike & Price \\
\hline Feb & 450 & 1.15 \\
\hline Feb & 480 & 2.7 \\
\hline Feb & 510 & 8.4 \\
\hline Feb & 540 & 23.9 \\
\hline Mar & 420 & 1.35 \\
\hline Mar & 450 & 3.1 \\
\hline Mar & 510 & 13.25 \\
\hline Apr & 300 & 0.25 \\
\hline Apr & 480 & 9.45 \\
\hline
\end{tabular}

Jan 8, 2010 Asset price : 528

Risk-free rate r : $0.149 \%$

\begin{tabular}{|c|c|c|}
\hline Expiration & Strike & Price \\
\hline Feb & 450 & 0.65 \\
\hline Feb & 480 & 2 \\
\hline Feb & 510 & 5.7 \\
\hline Mar & 450 & 2.1 \\
\hline Mar & 480 & 4.3 \\
\hline Apr & 360 & 0.55 \\
\hline Apr & 420 & 2.11 \\
\hline
\end{tabular}

Jan 13, 2010 Asset price : 528

Risk-free rate r : $0.152 \%$

\begin{tabular}{|c|c|c|}
\hline Expiration & Strike & Price \\
\hline Feb & 450 & 0.75 \\
\hline Feb & 480 & 1.72 \\
\hline Feb & 510 & 5 \\
\hline Feb & 540 & 17.4 \\
\hline Mar & 360 & 0.35 \\
\hline Mar & 420 & 1.2 \\
\hline Mar & 540 & 23.2 \\
\hline
\end{tabular}


Jan 14, 2010 Asset price : 530

Risk-free rate r : $0.137 \%$

\begin{tabular}{|c|c|c|}
\hline Expiration & Strike & Price \\
\hline Feb & 450 & 0.7 \\
\hline Feb & 480 & 1.4 \\
\hline Feb & 510 & 4.6 \\
\hline Feb & 540 & 16.4 \\
\hline Mar & 330 & 0.15 \\
\hline Mar & 360 & 0.2 \\
\hline Mar & 420 & 0.9 \\
\hline Mar & 450 & 1.85 \\
\hline Mar & 480 & 3.83 \\
\hline Mar & 510 & 8.4 \\
\hline Mar & 540 & 21 \\
\hline Apr & 480 & 6.2 \\
\hline
\end{tabular}

Jan 20, 2010 Asset price : 525

Risk-free rate r: $0.14 \%$

\begin{tabular}{|c|c|c|}
\hline Expiration & Strike & Price \\
\hline Feb & 300 & 0.05 \\
\hline Feb & 450 & 0.55 \\
\hline Feb & 480 & 1.45 \\
\hline Feb & 510 & 4.8 \\
\hline Feb & 540 & 19.6 \\
\hline Feb & 600 & 80.34 \\
\hline Mar & 390 & 0.55 \\
\hline Mar & 420 & 0.98 \\
\hline Mar & 450 & 1.85 \\
\hline Mar & 480 & 4.5 \\
\hline Mar & 510 & 9.7 \\
\hline Mar & 540 & 25.28 \\
\hline
\end{tabular}

Jan 15, 2010 Asset price : 524

Risk-free rate r : $0.149 \%$

\begin{tabular}{|c|c|c|}
\hline Expiration & Strike & Price \\
\hline Feb & 420 & 0.4 \\
\hline Feb & 450 & 0.75 \\
\hline Feb & 480 & 1.9 \\
\hline Feb & 510 & 5.7 \\
\hline Feb & 540 & 20.5 \\
\hline Mar & 420 & 0.8 \\
\hline Mar & 450 & 2.3 \\
\hline Mar & 480 & 4.7 \\
\hline Mar & 510 & 10.5 \\
\hline Mar & 540 & 26.05 \\
\hline Apr & 480 & 7.5 \\
\hline Apr & 540 & 29.2 \\
\hline
\end{tabular}

Jan 21, 2010 Asset price : 514

Risk-free rate r : $0.13 \%$

\begin{tabular}{|c|c|c|}
\hline Expiration & Strike & Price \\
\hline Feb & 360 & 0.08 \\
\hline Feb & 450 & 1.25 \\
\hline Feb & 480 & 3.1 \\
\hline Feb & 510 & 9.6 \\
\hline Mar & 300 & 0.1 \\
\hline Mar & 420 & 1.17 \\
\hline Mar & 450 & 2.9 \\
\hline Mar & 480 & 6.5 \\
\hline Mar & 510 & 15.4 \\
\hline Mar & 540 & 29.5 \\
\hline Apr & 360 & 0.7 \\
\hline Apr & 390 & 1.3 \\
\hline Apr & 420 & 2.6 \\
\hline Apr & 450 & 4.1 \\
\hline Apr & 480 & 6.62 \\
\hline May & 480 & 13.2 \\
\hline
\end{tabular}

Jan 19, 2010 Asset price : 530

Risk-free rate r : $0.137 \%$

\begin{tabular}{|c|c|c|}
\hline Expiration & Strike & Price \\
\hline Feb & 420 & 0.21 \\
\hline Feb & 450 & 0.45 \\
\hline Feb & 480 & 1.15 \\
\hline Feb & 510 & 3.7 \\
\hline Mar & 420 & 1 \\
\hline Mar & 480 & 3.3 \\
\hline Mar & 510 & 8.1 \\
\hline Mar & 540 & 19.9 \\
\hline Apr & 510 & 11.91 \\
\hline May & 300 & 0.25 \\
\hline May & 420 & 3.4 \\
\hline
\end{tabular}

Jan 22, 2010 Asset price : 502

Risk-free rate r : $0.124 \%$

\begin{tabular}{|c|c|c|}
\hline Expiration & Strike & Price \\
\hline Feb & 390 & 0.4 \\
\hline Feb & 420 & 1.4 \\
\hline Feb & 450 & 2.8 \\
\hline Feb & 480 & 6.2 \\
\hline Feb & 510 & 15.9 \\
\hline Feb & 540 & 38 \\
\hline Mar & 300 & 0.1 \\
\hline Mar & 360 & 0.85 \\
\hline Mar & 390 & 1.6 \\
\hline Mar & 420 & 3 \\
\hline Mar & 450 & 5.6 \\
\hline Mar & 480 & 10.3 \\
\hline Mar & 510 & 21.9 \\
\hline Mar & 540 & 39.4 \\
\hline Mar & 570 & 60.37 \\
\hline Apr & 420 & 4.6 \\
\hline Apr & 450 & 7.11 \\
\hline Apr & 480 & 13.1 \\
\hline Apr & 510 & 25.7 \\
\hline Apr & 540 & 38.3 \\
\hline
\end{tabular}


Jan 25, 2010 Asset price : 505

Risk-free rate r : $0.134 \%$

\begin{tabular}{|c|c|c|}
\hline Expiration & Strike & Price \\
\hline Feb & 420 & 0.95 \\
\hline Feb & 450 & 1.9 \\
\hline Feb & 480 & 4.8 \\
\hline Feb & 510 & 14 \\
\hline Feb & 540 & 34.86 \\
\hline Mar & 330 & 0.15 \\
\hline Mar & 420 & 2.5 \\
\hline Mar & 450 & 4.47 \\
\hline Mar & 480 & 9.2 \\
\hline Mar & 510 & 19.6 \\
\hline Mar & 540 & 40.2 \\
\hline Apr & 420 & 3.7 \\
\hline Apr & 480 & 11.6 \\
\hline \multicolumn{2}{|c}{}
\end{tabular}

Jan 28, 2010 Asset price : 500

Risk-free rate r : $0.145 \%$

\begin{tabular}{|c|c|c|}
\hline Expiration & Strike & Price \\
\hline Feb & 420 & 0.55 \\
\hline Feb & 450 & 1.3 \\
\hline Feb & 480 & 4.5 \\
\hline Feb & 510 & 16 \\
\hline Mar & 360 & 0.3 \\
\hline Mar & 420 & 1.8 \\
\hline Mar & 450 & 4.6 \\
\hline Mar & 480 & 9.2 \\
\hline Mar & 510 & 20.6 \\
\hline Mar & 540 & 42 \\
\hline Apr & 420 & 3.2 \\
\hline Apr & 480 & 13.5 \\
\hline Apr & 510 & 23 \\
\hline May & 480 & 17.6 \\
\hline
\end{tabular}

Jan 26, 2010 Asset price : 503

Risk-free rate r : $0.142 \%$

\begin{tabular}{|c|c|c|}
\hline Expiration & Strike & Price \\
\hline Feb & 420 & 1.05 \\
\hline Feb & 450 & 1.35 \\
\hline Feb & 480 & 4.7 \\
\hline Mar & 420 & 1.7 \\
\hline Mar & 450 & 4.2 \\
\hline Mar & 480 & 9.16 \\
\hline Mar & 510 & 20.9 \\
\hline Mar & 540 & 42 \\
\hline Mar & 570 & 67.29 \\
\hline Apr & 360 & 0.9 \\
\hline Apr & 450 & 6.7 \\
\hline Apr & 480 & 13 \\
\hline Apr & 510 & 24 \\
\hline Apr & 540 & 44.1 \\
\hline
\end{tabular}

Jan 29, 2010 Asset price : 495

Risk-free rate r : $0.142 \%$

\begin{tabular}{|c|c|c|}
\hline Expiration & Strike & Price \\
\hline Feb & 420 & 0.25 \\
\hline Feb & 450 & 1.6 \\
\hline Feb & 480 & 5.2 \\
\hline Feb & 510 & 20.4 \\
\hline Feb & 540 & 42.8 \\
\hline Mar & 360 & 0.3 \\
\hline Mar & 420 & 1.5 \\
\hline Mar & 450 & 3.3 \\
\hline Mar & 480 & 10.9 \\
\hline Mar & 510 & 25.6 \\
\hline Mar & 540 & 41 \\
\hline Apr & 420 & 3.3 \\
\hline Apr & 480 & 14.6 \\
\hline Apr & 510 & 27.1 \\
\hline Apr & 540 & 49.5 \\
\hline
\end{tabular}

Jan 27, 2010 Asset price : 506

Risk-free rate r : $0.157 \%$

\begin{tabular}{|c|c|c|}
\hline Expiration & Strike & Price \\
\hline Feb & 420 & 0.35 \\
\hline Feb & 450 & 1 \\
\hline Feb & 480 & 3.4 \\
\hline Feb & 510 & 12.6 \\
\hline Feb & 540 & 39.4 \\
\hline Mar & 330 & 0.2 \\
\hline Mar & 420 & 1.9 \\
\hline Mar & 450 & 4.1 \\
\hline Mar & 480 & 7.8 \\
\hline Mar & 510 & 19.3 \\
\hline Mar & 540 & 41.7 \\
\hline Apr & 480 & 11.25 \\
\hline
\end{tabular}

Feb 01, 2010 Asset price : 501

Risk-free rate r : $0.157 \%$

\begin{tabular}{|c|c|c|}
\hline Expiration & Strike & Price \\
\hline Mar & 420 & 1.35 \\
\hline Mar & 450 & 3.1 \\
\hline Mar & 480 & 8.1 \\
\hline Mar & 510 & 19.8 \\
\hline Apr & 420 & 3.2 \\
\hline Apr & 510 & 24 \\
\hline May & 420 & 4.7 \\
\hline May & 480 & 16.5 \\
\hline
\end{tabular}


Feb 02, 2010 Asset price : 508 Risk-free rate r : $0.165 \%$

\begin{tabular}{|c|c|c|}
\hline Expiration & Strike & Price \\
\hline Mar & 300 & 0.05 \\
\hline Mar & 420 & 1.15 \\
\hline Mar & 450 & 2.14 \\
\hline Mar & 480 & 5.8 \\
\hline Mar & 510 & 15.09 \\
\hline Mar & 540 & 37.6 \\
\hline Apr & 480 & 9.24 \\
\hline Apr & 510 & 19.5 \\
\hline Apr & 540 & 39.8 \\
\hline
\end{tabular}

Feb 03, 2010 Asset price : 505 Risk-free rate r : $0.165 \%$

\begin{tabular}{|c|c|c|}
\hline Expiration & Strike & Price \\
\hline Mar & 420 & 0.75 \\
\hline Mar & 450 & 2.1 \\
\hline Mar & 480 & 6 \\
\hline Mar & 510 & 16.02 \\
\hline Apr & 360 & 0.55 \\
\hline Apr & 450 & 4.7 \\
\hline Apr & 510 & 20.5 \\
\hline May & 480 & 14 \\
\hline
\end{tabular}

Feb 04, 2010 Asset price : 490 Risk-free rate r : $0.152 \%$

\begin{tabular}{|c|c|c|}
\hline Expiration & Strike & Price \\
\hline Mar & 360 & 0.35 \\
\hline Mar & 420 & 1.84 \\
\hline Mar & 450 & 4.83 \\
\hline Mar & 480 & 11.8 \\
\hline Mar & 510 & 27 \\
\hline Mar & 540 & 49.4 \\
\hline Mar & 570 & 80.7 \\
\hline Apr & 420 & 3.3 \\
\hline Apr & 450 & 7.33 \\
\hline Apr & 480 & 15.8 \\
\hline Apr & 510 & 29.2 \\
\hline Apr & 540 & 51.6 \\
\hline May & 480 & 20.5 \\
\hline
\end{tabular}

Feb 05, 2010 Asset price : 491

Risk-free rate r : $0.152 \%$

\begin{tabular}{|c|c|c|}
\hline Expiration & Strike & Price \\
\hline Mar & 360 & 0.35 \\
\hline Mar & 420 & 3.1 \\
\hline Mar & 450 & 6.7 \\
\hline Mar & 480 & 11.4 \\
\hline Mar & 510 & 26.8 \\
\hline Mar & 570 & 89.2 \\
\hline Apr & 420 & 4.3 \\
\hline Apr & 450 & 10.55 \\
\hline Apr & 480 & 17.2 \\
\hline May & 420 & 6.6 \\
\hline May & 480 & 21.6 \\
\hline
\end{tabular}

